\title{
Analysis of Circumferential Groove Casing Treatment on Axial Compressor
}

\author{
Tao YI, Fang XIE ${ }^{\mathrm{a}^{*}}$, De-Jun LIU, Tian-Ye JI \\ Xi'an Institute of High Technology, Xi'an, 710025, PR China \\ afangxiejiajia@163.com \\ ${ }^{*}$ Corresponding author
}

Keywords: axial compressor, casing treatment, circumferential groove, leakage flow.

\begin{abstract}
As an effective method to extend the stability range of axial compressor, circumferential groove casing treatment has always been the focus of researchers. This paper analyze the stall margin improvement of casing treatment and the flow field in axial compressor. Slot type and circumferential casing treatment are compared to conduct the development situation of optimized casing structure. We introduce a new casing treatment which can extend the stability range and improve efficiency of axial compressor. It turned out that keeping both pressure ratio and efficiency and improving the stall margin of compressor is the most important goal via designing reasonable tip clearance and circumferential groove casing treatment. The design principles can be used as a reference for the industrial sector.
\end{abstract}

\section{Introduction}

As knowing the tip clearance flow of compressor deeply, domestic and aboard scholars hope to improve functions of boosters by controlling clearance tip flow and propose casing treatment, tip jet, plasma actuation, sweeping-curved rotor, geometry patching of moving tip blades and other a series of methods based on a deal of researches. Led by Chu Wuli, this research group used methods of experiment and numerical simulation to explore casing treatment mechanisms and structure optimization and proposed certain new casing treatment forms. Accordingly, it is necessary to conclude recent development situation on casing treatment

\section{Stability Enhancement Effect and Mechanisms on Casing Treatment}

In recent years, domestic and overseas scholars explore the casing treatment stability enhancement effect deeply. Earlier research indicated that circumferential groove could not reduce efficiency but realize stability enhancement effect in the range of $10 \%$ [1]. But recent research found that stability enhancement effect of circumferential groove was related with stall ways of compressors, groove position, groove depth, groove amount closely and can reduce efficiency of compressors under most situations. Van[2] reckoned that stall ways of compressors are essential to stability enhancement. Because loading blade roots caused stall, circumferential grooves were not able to improve stall margin of compressors at all. In 2009, Houghton[3] installed circumferential single grooves on various positions of an acyclic low speed compressor. He found that stall margin improvement was largest at the positions of $8 \%$ or $50 \%$ axial chord away from the front blade, while tall margin improvement was least at the position of $18 \%$ axial chord away from the leading and trailing edge blade. If grooves were located on $8 \%$ axial chord away from the front blade, groove airflow would experience interaction with high-energy near casing intensively so that apex leakage flow, trace of apex leakage vortex and blade load would be changed and brought loss which reduced overall efficiency. Houghton tested axial velocity of compressor rotors which were at the exit flow field. He proposed that circumferential grooves influenced axial velocity of compressor rotors, in other words, blocking conditions in the channel was not improved by adding grooves. Wilk[4] found that casing treatment improved the stability margin of compressors, but it caused the efficiency reduction to varying degrees. Led by $\mathrm{Chu} \mathrm{Wuli}$, this research group explored groove or 
seam casing treatment stability enhancement effect and mechanism more deeply[5]. They reckoned that circumferential grooves reduced the driving force of apex leakage flow locomotion and weaken negative effect of clearance leakage flow. Because there exited pressure gradient between upstream and downstream of slots and low-energy fluid was inhaled by reflux oil which formed in the slots or overdrove to slot upstream under differential pressure, axial slots could enhance stability. But some scholars [6] reckoned that casing treatment could restrain the development of blade suction side of trailing edge separation zone. Groove depth was 7 to 8 times than the height of blade tip seam could improve stability margin of compressors and more amount or less wide grooves could better work.

\section{Groove or Seam Casing Treatment Structure Optimization}

The method of using circumferential grooves to treat casing was the earliest and most be widely used. Optimizing stability enhancement mechanisms or structure was the mainly work at present. The research group had done considerable research on slotting position, slotting numbers, slotting depth and slotting width. The experimental and numerical results indicated that slotting width in the same circumstances; the most slotting numbers made the stability enhancement effect best, while the least slotting numbers made the stability effect worst[7]. Liu Chuanle[8] reckoned various axial positions of circumferential grooves caused diverse damage. Under exact circumferential groove arrangement circumstances, changing circumferential groove depth could make 'optimal circumferential groove depth. Zhang Haoguang[9] explored the slotting positions of circumferential grooves deeply through experimental and numerical simulation. And he reckoned that axial positions which were at the middle of blade tip could have the best influence on stability enhancement effect. Moving axial positions forward had the second best influence on stability enhancement effect, while moving axial positions backward had the worst influence on stability enhancement effect. Based on three elements of slotting depth, slotting width and center offset, $\mathrm{Wu}$ Yanhui[10] designed nine circumferential grooves casing treatment structure and did orthogonal experiments in order that finding an optimal combination of slotting depth, slotting width and center offset to have the greatest effect on stall margin improvement. And finding an optimal combination had a great influence on stall margin for circumferential groove width. Under low rpm circumstance, circumferential groove depth had the worst influence on stall margin and circumferential groove center offset had the worst influence on stall margin under high rpm circumstance. For further wider stability margin, Mi Pan[11] did numerical simulation for axial tilt circumferential grooves. He found that stability margin could be gained if the angle of top rake was $63.4^{\circ}$ for circumferential grooves. Further analyze flow field, circumferential groove fore rake could reduce flow losses, enhance circumferential carriage capacity and reduce radial carriage capacity of grooves, which made stability enhancement effect better. Li Xiangjun[12] studied stability enhancement effect of circumferential shallow grooves for treating casings, and shallow grooves which were located various axial positions treat casings. Circumferential shallow grooves could enhance stability of compressors validly, but that capacity could change compressors' stall mechanism apparently by dong experiments. Chu Wuli and Shen Kai $\left[13^{]}\right.$studied the coupling effect between entry guide vanes and blade angle slots to treat casings through a deal of experiments and concluded that coupling effect can make wider working stability margin. Summarizing structure optimization of groove or seam casing treatment from present research achievements, the key geometry parameters was as following:

(1) Existing experimental and numerical results indicate: moving axial positions forward had the second best influence on stability enhancement effect, while moving axial positions backward had the worst influence on stability enhancement effect;

(2) Existing optimal groove width range makes casings' key parts had the largest projection area on tip chord could make circumferential grooves play a better role. Compared with wide-slot casing treatment, narrow-groove casing treatment could enhance blade inlet angle in the blade tip zone and inlet axial velocity;

(3) Existing experimental and numerical results indicated: If the ratio between groove depth and blade height was from 0.155 to 0.207 , casing treatment would gain optimal stability enhancement 
effect;

(4) A deal of casing treatment experiments indicated that the near stall pressure ratio was less than solid casings'. The wide or deep slot circumferential grooves had high near stall pressure ratio. Abundant casing treatment's peak efficiency was lower than solid casings' and only shallow slots' peak efficiency was higher than solid casings'.

Based on the above-mentioned research situation, the research group reckoned that it was necessary to study the coupling effect between tip clearance and circumferential grooves and needed to more intensive studies on coupling effect in order to gain more valid stability enhancement methods. Domestic and aboard scholars also lay much attention on that field. Houghton found that the stability enhancement was superior when circumferential single grooves were located on the $50 \%$ axial chord position. When the single-groove structure became multiplegroove structure, stability enhancement margin of multiple-groove structure would not increase linearly as single-groove structure. Sakuma [14] reckoned that circumferential single groove could improve stability margin, but the position design was quite essential. Liu Le[15] reckoned front grooves can restrain unsteady leakage flow, while the influence of back-end single grooves was not apparent through experiment research. Based on the above mentioned, the author reckoned circumferential single groove position was essential and it was necessary to study stall mechanism of compressors in order to decide position of grooves.

\section{Development of New Stability Enhancement Synergy of Casing Treatment}

Except for structure optimization of traditional casing treatment, more casing treatment methods were arising. Sun Dakun[16] designed an unsteady casing treatment method through changing chutes and air chambers to adjust the surface impedance conditions' parameters. And he used a series of experiment research to study that casing treatment method. By comprising two situations of smooth walls and casing treatment, he convinced that treating casings could restrain non-linear development of stall inception. And no matter what kinds of portent wave could be restrained validly through vortex interaction. It could restrain low-frequency perturbation wave development and postpone rotating stall accidents so that stability enhancement could be realized. Compared with traditional casing treatment, that new-style casing treatment possessed low punching rate, low efficiency damage, compact structure, active adjustment, and other merits which could meet stability enhancement conditions in various working states. Xiong Shan[17] did experimental research on stability enhancement effect of casing treatment under inlet distortion situation and proved that casing treatment had excellent stability enhancement effect for various degrees of distortion. Dong $\mathrm{Xu}[18]$ did experimental research on inlet rotating distortion about casing treatment. Experimental results indicated that new-style casing treatment would have predominant stability enhancement effect under inlet rotating distortion conditions. Hathaway[19] proposed a new casing treatment form in use of numerical simulation. That casing used air injection approach and bled air to postpone compressors' stall and adjusted its injection rate or air-blowing quantity according to compressors' operating conditions. Under little efficiency damage situation, stall margin would enhance according to numerical simulation. Strazisar[20] used self-recirculating casing to do experiments on high-load and transonic speed compressors. Experimental results showed that self-recirculating casing enhances stability of high load compressors. Zhang Haoguang[21] studied mechanisms which could improve compressors' properties. He reckoned that the casing treatment restrained development of clearance backflow and amplifies airflow inlet angle in tip blade, outlet angle and axial inlet velocity. That method also restrained boundary layer separation on blade back and development of clearance leakage vortex limitation in blade tip area contributing to reduce flow damage. Cyril Guine[22] saw 1.5 class compressors as object of study. He used the method of unsteady constant value simulation to study self-recirculating casing treatment effect under various tip clearance conditions. Results indicated that the efficiency of selfrecirculating casing treatment was not altered under design conditions whether in maintaining clearance design or adding clearance design, but the stall could be postponed and stability could be enhanced. It could also cause negative effect in stability if tip clearance was reduced. 


\section{Conclusion}

Based on studies above mentioned, there existed some problems:

(1)Although studies of circumferential grooves had researched deeply, studies of single circumferential grooves hadn't studied deeply. Positions of single circumferential grooves were essential, it was necessary to study compressor stall mechanism deeply for achieving excellent design effect. It was uncommon to find relevant studies on design optimization of single circumferential grooves' depth and width;

(2) Because there was negative effect which influenced stability, it was necessary to study tip clearance and casing treatment coupling effect. As a result, how to design tip clearance and single circumferential grooves was an essential direction in order to maintain step-down ratio or efficiency and improve stability margin. And the design principle could provide important reference for industry field.

\section{References}

[1] Liu Zhiwei;Zhang Changsheng. The circumferential groove casing treatment on a number of observations [J]. Journal of Northwestern Polytechnical University.1985,3(2):202 217

[2] Van de Wyer N Farkas B Desset J. Experimental Investigation of the Steady and Unsteady Flow Field in A Single Stage Low Pressure Axial Compressor with A Circumferential Groove Casing Treatment[R]. ASME paper No. GT2010-23474, 2010.

[3] Houghton T, Day I. Enhancing the Stability of Subsonic Compressors Using Casing Grooves [R]. ASME paper No.r GT2009-59210, 2009.

[4] Wilke I, Kau H P. A numerical investigation of the flow mechanisms in a HPC front stage with axial slots[R]. ASME Paper GT2003-38481, 2004.

[5] ZHANG Hao-guang, CHU Wu-li. Investigation of the flow mechanisms of affecting compressor performance with axial skewed slots casing treatment [J].Journal of Propulsion Technology. 2010, 31(5):555 562.

[6] Huang Xudong. Numerical Investigation of the Stall Mechanism and Casing Treatment of Transonic Compressor [D].Beijing: Tsinghua University, 2010.

[7] Chu Wuli, Zhang haoguang, Wu Yanhui. Effects of grooved number of grooved case treatment on stall margh [J]. Journal of Propulsion Technology.2008, 29(5): 514 598.

[8] Liu chuanle, Chu Wuli, Zhang Haoguang,. The Influence of Circumferential Groove Casing Treatment on Compressor Stability [J]. Compressor, Blower \& Fan Technology.2011(2):6 11

[9] Zhang Haoguang. Investigation of experimental and numerical casing processing on Axial flow compressor 1 [D].Xian: Northwestern Polytechnical University, 2008.

[10]Wu Yanhu, Zhang Haoguang. Orthogonal experimental investigation of geometry structure of grooved casing treatment [J]. Journal of Aerospace Power. 2009, 24(4): 825 829

[11]Mi Pan, Chu Wuli. Research on the Effects of Axial Inclined Circumferential Grooves Casing Treatment on Stability Extension of Compressors [J]. Compressor, Blower \& Fan Technology. 2011(6):3 8

[12]Chu Wuli, ShenKai. Experiment I investigation and M echanism Analysis of I nteraction of Variable I nlet GuideVanesand Axial Skewed SiotsCasing Tr eat m ent [J]. Journal of Propulsion Technology. 2014(9): 1209 1215.

[13]Sakuma Y, Watanabe T. Numerical Analysis of Flow in A Transonic Compressor with a Single Circumferential Casing Groove: Application to Two Different Compressor Rotors[R].ASME paper No.GT2014-26691, 2014. 
[14]LIU Len, NAN Xi. Investigation of Stability Enhancing Methods Based on Circumferential Single Groove on Low Speed Axial Compressor [J]. Journal of Engineering Thermophysics.2012, 33(12): 2063 2066.

[15]SU N Da kun, H OU Rui-w ei, SU N Xiao-fend. An experimental investigation of rotating stall margin improvement with unsteady casing treatments [J]. Journal of Aerospace Power.2008, 23(4): $825 \sim 829$.

[16]XIONG Shan; SUN Dakun; SUO Qiuling. Experimental Investigation of Novel Casing Treatment on Stall Margin Enhancement under Inlet Distortion [J]. Acts Aeronautics et Astronautics Sinica.2014, 34(12):2692 2700.

[17]DONG Xu; LIU Xiaohua; SUN Dakun. Experimental Investigation of Stall Margin Enhancement Using Novel Casing Treatment under the Rotating Inlet Distortion [J]. Acts Aeronautics et Astronautics Sinica.2014, 35(9): 2411 2425.

[18]Hathaway. Michael D, Self-Recalculating Casing Treatment Concept for Enhanced Compressor Performance[R]. ASME paper No.GT-2002-30368, 2002.

[19]Strazisar.J. Anthony. Compressor Stall Control through Endwall Recirculation[R].ASME paper No.GT- 2004-54295.

[20]ZHANG Hao-guang; WANG Yun-peng; WU Jun, Investigation on Mechanism of Performance Improvement for Compressor with New Type of Casing Treatment with Inlet Distortion [J]. .Journal of Propulsion Technology. 2014, 35(8): 1040 1047. 